\title{
Targeting Mutant (V600E) B-Raf in Melanoma Interrupts Immunoediting of Leukocyte Functions and Melanoma Extravasation
}

\author{
Shile Liang ${ }^{1}$, Arati Sharma ${ }^{3}$, Hsin-Hsin Peng ${ }^{2}$, Gavin Robertson ${ }^{3,4,5,6,7}$, and Cheng \\ Dong ${ }^{1,2,7}$ \\ ${ }^{1}$ Huck Institutes of the Life Sciences, The Pennsylvania State University, University Park, \\ Pennsylvania \\ ${ }^{2}$ Department of Bioengineering, The Pennsylvania State University, University Park, Pennsylvania \\ ${ }^{3}$ Department of Pharmacology, The Pennsylvania State University College of Medicine \\ ${ }^{4}$ Department of Pathology, The Pennsylvania State University College of Medicine \\ ${ }^{5}$ Department of Dermatology, The Pennsylvania State University College of Medicine \\ ${ }^{6}$ The Foreman Foundation for Melanoma Research \\ ${ }^{7}$ The Penn State Melanoma Therapeutics Program, Hershey, Pennsylvania
}

\section{Abstract}

Polymorphonuclear neutrophils (PMN) facilitate melanoma cell extravasation under dynamic flow conditions by the binding of intercellular adhesion molecule-1 (ICAM-1) on melanoma cells to $\beta_{2}$ integrins on PMNs, which is mediated by endogenously produced chemokine interleukin 8 (IL-8) from the tumor microenvironment. However, little is known about the role of $B-R a f$, the most mutated gene in malignant melanomas, in this process. In this study, we investigated the functional importance of $B$-Raf in melanoma extravasation by using short interfering RNA to reduce expression/activity of mutant ${ }^{V 600 E} B$-Raf in melanoma. Results indicated that knockdown of mutant ${ }^{V 600 E} B$-Raf inhibited melanoma cell extravasation in vitro and subsequent lung metastasis development in vivo. Mechanistic studies showed that inhibition of ${ }^{V 600 E} B$-Raf significantly reduced the constitutive secretion of IL-8 from melanoma cells as well as the capacity of endogenous IL-8 production from the melanoma-PMN microenvironment. Furthermore, a reduction in ICAM-1 expression on melanoma cells was detected following mutant ${ }^{V 600 E} B$-Raf knockdown. Together, these results suggest that targeting mutant ${ }^{V 600 E} B$-Raf reduces melanoma cell extravasation by decreasing IL-8 production and interrupting ICAM-1- $\beta_{2}$ integrin binding of melanoma cells to the endothelium mediated by PMNs in the microcirculation, which provides a rationale and mechanistic basis for targeting mutant ${ }^{V 600 E} B$-Raf to inhibit melanoma extravasation and subsequent metastasis development.

\section{Introduction}

Melanoma is a skin cancer characterized by abnormal proliferation of melanocytes that invade the basement membrane. Once melanoma metastasizes, it is notoriously resistant to diverse

(C)2007 American Association for Cancer Research.

Requests for reprints: Cheng Dong, Department of Bioengineering, The Pennsylvania State University, 233 Hallowell Building, University Park, PA 16802. Phone: 814-865-8091; Fax: 814-863-0490; E-mail: cxd23@psu.edu. 
chemotherapeutic agents, and for many patients, prognosis is poor. Consequently, the search for novel antimelanoma agents continues. Studies have shown that mutation in B-Raf, a glutamic acid for valine substitution at codon 600 (V600E) in exon 15, happens in $\sim 60 \%$ of melanomas (1-4). $B$-Raf encodes a RAS-regulated kinase that mediates cell growth and malignant transformation (5). B-Raf mutation is implicated in constitutive activation and hyperactivation of downstream proteins in the signaling cascade that promotes melanoma adhesion, migration, and proliferation $(1,4,6,7)$.

Melanoma metastasis requires that tumor cells detach from a primary site and invade the surrounding stroma, survive immune defenses and turbulence of the blood circulation after invading into the circulatory system, extravasate through the endothelial lining of blood vessels, and finally form a new colony in the surrounding tissue. Human polymorphonuclear neutrophils (PMN), which compose 50\% to 70\% of circulating leukocytes, have been shown under certain circumstances to promote tumor adhesion and transendothelial extravasation $(8,9)$. Miele et al. $(10)$ reported that a dose- and time-dependent increase in surface expression of intercellular adhesion molecule-1 (ICAM-1) was found in human malignant melanoma cells. Furthermore, binding between ICAM- 1 on malignant melanoma cells and $\beta_{2}$ integrins (e.g., CD11a/CD18, LFA-1 and CD11b/CD18, Mac-1) found on PMNs mediates melanoma extravasation through the endothelium (Fig. 1; refs. 11,12). Endogenous interleukin 8 (IL-8) secreted by melanoma cells also increases $\beta_{2}$ integrin expression on PMNs and modulates PMN-facilitated melanoma extravasation (12). IL-8 protein production requires the activation of nuclear transcription factor $\mathrm{\kappa B}$ (NF- $\mathrm{\kappa B})$, NF-IL-6 (C/EBP), or activator protein-1 $(13,14)$. However, little is known about the regulation of IL-8 production and of interactions between PMN and melanoma by mutant ${ }^{V 600 E} B$-Raf in melanoma cells, which may, in turn, influence melanoma extravasation.

In the present study, we evaluated the role played by mutant ${ }^{V 600 E} B$-Raf in melanoma extravasation and metastasis development. Short interfering RNA (siRNA) targeting mutant ${ }^{V 600 E} B$-Raf was used to reduce expression and/or activity of mutant ${ }^{V 600 E} B$-Raf in melanoma cells. Results suggest that inhibition of mutant ${ }^{V 600 E}$ BRaf reduced melanoma metastasis in vivo by decreasing melanoma cell extravasation. The mechanism for reduced melanoma extravasation was due to decreased ICAM-1 expression, which occurred with decreased mutant ${ }^{V 600 E} B$-Raf expression levels. In addition, constitutive secretion of the chemokine IL-8 from melanoma cells, as well as the capacity of increased IL-8 production from a melanoma-PMN microenvironment, was significantly decreased following inhibition of mutant ${ }^{V 600 E} B$-Raf. Coupling these observations with previous studies showing that endogenously produced IL- 8 and ICAM-1- $\beta_{2}$ integrin binding mediate melanoma extravasation $(11,12)$ provides a rationale and mechanistic basis for targeting mutant ${ }^{V 600 E} B$ $R a f$ to inhibit melanoma extravasation and subsequent metastasis development.

\section{Materials and Methods}

\section{Cell preparations}

The human melanoma cell lines $1205 \mathrm{Lu}$ and UACC 903M containing high levels of mutant ${ }^{V 600 E} B$-Raf $(15,16)$ were grown in DMEM (Invitrogen) supplemented with $10 \%$ fetal bovine serum (FBS; Invitrogen). Green fluorescent protein (GFP)-tagged versions of $1205 \mathrm{Lu}$ and UACC 903M were used for metastasis assays. EI cells are fibroblast L-cells transfected to express human E-selectin and ICAM-1 (provided by Dr. Scott Simon, University of California Davis, Davis, CA) and were maintained in culture as previously described (17).

Use of PMNs was approved by the Pennsylvania State University Institutional Review Board (\#19311). Fresh human blood was collected from healthy adults by venipuncture. PMNs were isolated using a Histopaque (Sigma) density gradient as described by the manufacturer and 
kept at $4{ }^{\circ} \mathrm{C}$ in Dulbecco's PBS containing $0.1 \%$ human serum albumin until use. Cell preparations were $99.5 \%$ pure PMNs, confirmed by a Diff-Quick stain (Dade Behring, Inc.). In the cases of PMN and melanoma cell coculture, PMNs resuspended in RPMI 1640 supplemented with 5\% FBS were added onto the confluent melanoma cell lines (1:1 ratio) directly and cocultured for $4 \mathrm{~h}$. Ninety-five percent melanoma cells stay alive after 4-h contact culture with PMNs (data not shown).

\section{In vitro and in vivo siRNA studies}

SiRNA (100 pmol) was introduced into $1 \times 10^{6} 1205 \mathrm{Lu}$ or UACC 903M via nucleofection with an Amaxa Nucleofector using Solution R/program K-17 as previously described (15). The resultant transfection efficiency following nucleofection was $>90 \%$ (15) and knockdown of mutant ${ }^{V 600 E}$ B-Raf protein in $1205 \mathrm{Lu}$ and UACC 903M melanoma cells persisted beyond 8 days in culture compared with control cells nucleofected with scrambled siRNA (18). Specificity of using this siRNA approach to knock down mutant ${ }^{V 600 E}$ B-Raf has previously been described $(18,19)$. Duplexed stealth siRNA (Invitrogen) was used for these studies. The sequence for each respective siRNA is as follows: scrambled siRNA,

AAUUCUCCGAACGUGUCACGUGAGA; MUT B-Raf, GGUCUAGCUACAGAGAAAUCUCGAU; scrambled siRNA for ICAM- 1 , GCTGTGCACGTATGAGATA; ICAM-1, GCTGACGTGTGCAGTAATA.

Animal experimentation was done according to protocols approved by the Institutional Animal Care and Use Committee at The Pennsylvania State University College of Medicine. SiRNA $(100 \mathrm{pmol})$ was nucleofected into GFP-tagged $1205 \mathrm{Lu}$ or UACC $903 \mathrm{M}$ cells, which were then replated in culture dishes. Thirty-six hours later, $1 \times 10^{6}$ of $1205 \mathrm{Lu}$ or $0.5 \times 10^{6}$ of UACC $903 \mathrm{M}$ in $0.2-\mathrm{mL}$ HBSS were injected i.v. into the lateral tail veins of nude mice. The effect of siRNA knockdown in melanoma xenograft module can last up to day 17.5 (19). Mice were sacrificed 17 days later and lungs were analyzed for the presence of fluorescent tumors using a Nikon SMZ 1500 dissecting microscope with a Plan Apo 1.6× objective and fluorescence detection capabilities. Images of five random fields were photographed at a magnification of $\times 4.8$ from the ventral surface of each lung and the number of fluorescent tumors as well as area scored in pixels occupied by each tumor was quantified using IP lab imaging software (Scanalytics). Duplicate experiments consisting of eight animals were used per group.

\section{Colocalization of mouse PMN and human melanoma cell line}

Colocalization of mouse PMNs and human melanoma cells in lungs was used to verify interactions between cells and provide evidence of tethering of melanoma cells to the endothelium lining mediated by PMNs. Briefly, $1 \times 10^{6}$ GFP-tagged human metastatic melanoma cells $(1205 \mathrm{Lu})$ in $0.2 \mathrm{~mL}$ of HBSS were injected i.v. into the lateral tail veins of nude mice. After $24 \mathrm{~h}$, phycoerythrin-conjugated rat anti-mouse Gr-1 monoclonal antibody (BD PharMingen) at a concentration of $1 \mathrm{mg} / \mathrm{kg}$ of body weight in $100-\mu \mathrm{L}$ PBS was injected i.v. into the lateral tail veins of mice. Mice were sacrificed $2 \mathrm{~h}$ later and lungs analyzed using a Nikon SMZ 1500 dissecting microscope with fluorescence detection capabilities.

\section{ELISA determination}

At the end of coculture assays, cell-free supernatants were collected by centrifugation at 430 $\times g$ for 5 min and IL-8 was quantified by a sandwich ELISA following standard protocols. Primary and secondary antibody pairs were obtained from R\&D Systems. Standard human recombinant IL-8 was also obtained from R\&D Systems and a standard curve was included in each ELISA plate. Plates were read on a Packard Spectracount at $405 \mathrm{~nm}$ and the data analyzed using I-Smart Software. Intra-assay variation was typically $10 \%$ to $15 \%$. 


\section{Flow migration assay}

Flow migration assay was measured in a modified 48 -well chemotactic Boyden chamber consisting of a top and bottom plate separated by a gasket $(8,20)$. Before each experiment, a monolayer of EI cells was grown (typically $36 \mathrm{~h}$ after cell seeding) on sterile polyvinylpyrrolidone-free polycarbonate filters ( $8 \mu \mathrm{m}$ pore size; NeuroProbe) precoated with fibronectin $(30 \mu \mathrm{g} / \mathrm{mL}, 3 \mathrm{~h}$; Sigma). The center 12 wells of bottom plate were filled with soluble chemoattractant type IV collagen [100 $\mu \mathrm{g} / \mathrm{mL}$ in RPMI 1640/0.1\% bovine serum albumin (BSA); BD Biosciences] and surrounding control wells were filled with medium (RPMI $1640 / 0.1 \%$ BSA). Studies have shown that melanoma cells express $\alpha_{2} \beta_{1}$ integrin receptors for soluble collagen IV protein and migrate toward collagen IV stimulation $(21,22)$. Apparatus was assembled by placing filter on bottom plate followed by addition of a sealing gasket and top plate. The chamber was primed with $37^{\circ} \mathrm{C}$ medium to eliminate bubbles in the system. For migration assay, $5 \times 10^{5}$ melanoma cells only or PMNs together with melanoma cells ( $5 \times$ $10^{5}$ of each) were put in the chamber either under static conditions or under shear flow conditions for $4 \mathrm{~h}$ in a $37^{\circ} \mathrm{C}, 5 \% \mathrm{CO}_{2}$ incubator. To quantify migration, migrated cells were stained with Protocol Brand Hema3 solution (Fisher Scientific) and counted using an inverted microscope (Diaphot 330, Nikon) with NIH Image software (v. $\beta 4.0 .2$ ).

\section{Electromobility shift assay}

Nuclear extracts were prepared by lysing $10^{6}$ cells with $10 \%$ NP40 in buffer A $[10 \mathrm{mmol} / \mathrm{L}$ HEPES (pH 7.9), $10 \mathrm{mmol} / \mathrm{L} \mathrm{KCl,} 0.1 \mathrm{mmol} / \mathrm{L}$ EDTA, $0.1 \mathrm{mmol} / \mathrm{L}$ EGTA, $1 \mathrm{mmol} / \mathrm{L}$ DTT, $0.5 \mathrm{mmol} / \mathrm{L}$ phenylmethylsulfonyl fluoride (PMSF)] for $15 \mathrm{~min}$. Cells were then spun to separate nuclei from cytoplasmic component. Nuclear pellet was resuspended in buffer $\mathrm{C}$ [20 $\mathrm{mmol} / \mathrm{L}$ HEPES (pH 7.9), $0.4 \mathrm{~mol} / \mathrm{L} \mathrm{NaCl}, 1 \mathrm{mmol} / \mathrm{L}$ EDTA, $1 \mathrm{mmol} / \mathrm{L}$ EGTA, $1 \mathrm{mmol} / \mathrm{L}$ DTT, $1 \mathrm{mmol} / \mathrm{L}$ PMSF] for $15 \mathrm{~min}$. Annealed NF- $\mathrm{kB}$ binding site oligonucleotides (5'AGCTAAGGGACTTTCCGCTGGGGACTTTCCAGG-3' and 5'AGCTCCTGGAAAGTCCCCAGCGGAAAGTCCCTT-3') were end-filled with [ $\alpha_{-}{ }^{32} \mathrm{P}$ ] dCTP using bacterial Klenow fragment (Promega). The DNA probe was incubated with nuclear extracts in a reaction mixture containing dI-dC (GE Healthcare), $0.25 \mathrm{~mol} / \mathrm{L}$ HEPES ( $\mathrm{pH} 7.5$ ), $0.6 \mathrm{~mol} / \mathrm{L} \mathrm{KCl}, 9 \%$ glycerol, $1 \mathrm{mmol} / \mathrm{L} \mathrm{EDTA}, 0.75 \mathrm{~mol} / \mathrm{L} \mathrm{DTT}$, and $50 \mathrm{mmol} / \mathrm{L} \mathrm{MgCl}_{2}$ for $30 \mathrm{~min}$ at room temperature. A total of $0.5 \mu \mathrm{g}$ of anti-p50 or anti-p65 (NF- $\mathrm{kB}$, Santa Cruz Biotechnology) was added to the reaction mixture during the 30-min incubation to supershift complexes. The incubation mixtures were run on a $6 \%$ polyacrylamide gel at $130 \mathrm{~V}$ and the gel was dried and photographed.

\section{Statistics}

All results were shown as mean \pm SE unless otherwise stated. Student's $t$ test was used for pairwise comparisons and ANOVA was used for groupwise comparisons, followed by the appropriate post hoc test (Dunnett's, Tukey's, or Dunn's). Results were considered significant at $P<0.05$.

\section{Results}

\section{SiRNA-mediated inhibition of mutant ${ }^{V 600 E}$ B-Raf reduced melanoma metastases}

Because $B$-Raf is the most mutated gene in melanomas, it is an attractive therapeutic target to inhibit melanoma metastasis. To develop a more thorough understanding of its function in metastasis, siRNA was used to knockdown expression of mutant ${ }^{V 600 E} B$-Raf in the metastatic human melanoma cell lines $1205 \mathrm{Lu}$ and UACC 903M. Experimental metastasis development was studied by nucleofecting siRNA into melanoma cells, allowing recovery in culture for 1.5 days followed by i.v. injection of cells into the tail veins of nude mice. Seventeen days later, mice were euthanized and number and size of lung metastases scored. Figure $2 A$ shows that 
reducing expression/activity of mutant ${ }^{V 600 E} B$-Raf significantly decreased the metastatic potential of $1205 \mathrm{Lu}$ and UACC 903M cells. A significant 5- to 7-fold decrease in tumors $>1,500$ pixels was found following inhibition of ${ }^{V 600 E} B$-Raf versus control animals injected with cells nucleofected with scrambled siRNA (Fig. 2A, left). Similar results were observed in UACC $903 \mathrm{M}$ cells following inhibition of ${ }^{V 600 E} B$-Raf, with a 2- to 3-fold decrease in metastases $>1,500$ pixels compared with a scramble siRNA control (Fig. 2A, right).

Previous studies have shown that human PMN facilitates melanoma extravasation in vitro $(11,12)$. To investigate the interaction of melanoma cells with mouse PMNs, colocalization of green GFP-tagged melanoma cells and red phycoerythrin-tagged mouse PMNs in the lungs was studied. Results showed that green GFP-tagged 1205 Lu cells and red phycoerythrinlabeled PMNs can be detected microscopically in the lung stroma (Fig. 2B). More importantly, $\sim 13 \%$ melanoma cells and mouse PMNs were colocalized together, indicating that mouse PMNs are also facilitating melanoma cell adhesion to the endothelium, which could promote extravasation and thus metastases.

\section{Inhibiting mutant ${ }^{V 600 E} B$-Raf reduced melanoma cell ex-travasation}

Melanoma cell lung metastasis in vivo requires adhesion to and subsequent extravasation through the endothelial lining of lung vessels (23). To evaluate the role of mutant ${ }^{V 600 E} B$ $R a f$ in facilitating melanoma extravasation, $1205 \mathrm{Lu}$ cell extravasation was tested using an in vitro flow migration model (Fig. 3). Under static condition, melanoma only (without PMNs) migration was reduced by $25 \%$ following mutant ${ }^{V 600 E} B$-Raf inhibition (Fig. $3 A$ ). Under flow conditions of $4 \mathrm{dyn} / \mathrm{cm}^{2}$ when PMNs were not present, very few migrations of melanoma cells were observed and there were no migratory differences between control cells and those in which mutant ${ }^{V 600 E} B$-Raf had been inhibited (Fig. 3B, -Neutrophil). Previous studies have shown that PMNs facilitated melanoma adhesion and subsequent extravasation through an endothelial-like cell monolayer $(8,11,12,20)$. Our results confirmed that PMNs promoted 1205 Lu melanoma cell migration under flow conditions of $4 \mathrm{dyn} / \mathrm{cm}^{2}$ (Fig. $3 B,+$ Neutrophil) compared with melanoma cells alone (Fig. 3B,-Neutrophil). Furthermore, inhibition of mutant ${ }^{V 600 E} B$-Raf significantly retarded PMN-mediated melanoma cell extravasation by $\sim 2$ fold under flow conditions (Fig. 3B, +Neutrophil), indicating that inhibition of mutant ${ }^{V 600 E}$ B-Raf may affect interactions between PMNs and melanoma cells. These data showed that inhibition of mutant ${ }^{V 600 E} B$-Raf significantly retards melanoma extravasation across the endothelial-like cell monolayer, suggesting one potential mechanism for regulating metastasis development.

\section{Inhibiting mutant ${ }^{V 600}$ B-Raf reduced IL-8 production from a melanoma-PMN microenvironment}

Prior studies report that endogenously produced IL-8 mediate PMN-facilitated melanoma extravasation $(12,24)$. To evaluate the role played by mutant ${ }^{V 600 E} B-R a f$ in chemokine production from melanoma cells, which could affect melanoma cell extravasation, cells nucleofected with siRNA against mutant ${ }^{V 600 E} B$-Raf were examined for IL-8 production. Results showed that inhibition of mutant ${ }^{V 600 E} B$-Raf significantly reduced IL-8 production from melanoma cells alone. Specifically, IL-8 produced by control melanoma cells (untransfected melanoma and melanoma nucleofected with buffer only or scrambled siRNA) was $\sim 500 \mathrm{pg} / \mathrm{mL} / 10^{5}$ cells (Fig. $4 A$ ). However, inhibition of mutant ${ }^{V 600 E} B$-Raf resulted in $\sim 2-$ fold less IL-8 production (Fig. $4 A$ ). In addition, IL-8 production from the melanoma-PMN microenvironment was also inhibited after knockdown of mutant ${ }^{V 600 E} B-R a f$. Results showed that, following coculture of PMN and control melanoma cells, IL-8 production increased $~ 1.5-$ fold compared with the summed background levels of melanoma and PMN cultured alone (Fig. $4 B$ ). In contrast, approximately equivalent or slightly reduced levels of IL-8 were detected in the cocultures between PMN and melanoma cells (1205 Lu or UACC 903M) nucleofected with 
siRNA targeting mutant ${ }^{V 600 E}$ B-Raf (Fig. 4B). These data suggest that knockdown of mutant ${ }^{V 600 E} B$-Raf significantly reduced IL-8 production from melanoma cells alone and reduced the capacity of IL-8 production from the tumor microenvironment involving the immune system.

\section{Disruption of ICAM-1- $\beta_{2}$ integrin binding between melanoma and PMN following inhibition of mutant ${ }^{V 600 E_{B}-R a f}$ retarded melanoma extravasation}

Previous studies have shown that functional binding of ICAM-1 expressed on melanoma to $\beta_{2}$ integrin (LFA-1 and Mac-1) ligands expressed on PMNs caused cooperative tethering of PMN-melanoma aggregate to endothelium, which subsequently promotes melanoma extravasation $(8,12,24)$. To investigate the functional role of ICAM- 1 on melanoma to facilitate melanoma extravasation, melanoma cells were nucleofected with siRNA to reduce ICAM-1 expression, and extravasation under flow conditions was then measured. Flow cytometry showed that siRNA-mediated knockdown of ICAM-1 resulted in reduced expression of ICAM-1 (Fig. 5A). Flow migration results indicated that siRNA-mediated knockdown of ICAM-1 reduced melanoma cell extravasation significantly compared with the controls (Fig. $5 B$ ). These results suggested an important role for ICAM-1 in melanoma extravasation under flow conditions. Interestingly, inhibition of mutant ${ }^{V 600 E} B$-Raf reduced expression of ICAM-1 on melanoma cells to a similar level of that observed following siRNA ICAM-1 knockdown (Fig. 5A). In addition, extravasation of melanoma cells was reduced even more significantly following inhibition of mutant V600EB-Raf (Fig. 5B) compared with siRNA ICAM-1 knockdown cases, suggesting that inhibition of mutant ${ }^{V 600 E} B$-Raf has multiple effects other than reducing ICAM-1 expression.

Our results above have shown that inhibition of mutant ${ }^{V 600 E} B-R a f$ retarded IL-8 production in melanoma-PMN microenvironment. IL-8 has been shown to activate Mac-1 expression on PMNs $(25,26)$; we then investigated the effects of inhibiting mutant ${ }^{6600 E} B$-Raf on the expression of Mac-1 on PMNs. Results showed that Mac-1 expression on PMNs increased $\sim 2$ fold following coculture with control melanoma cells (Fig. 5C). In contrast, in the cases of PMN cocultured with $1205 \mathrm{Lu}$ cells following inhibition of mutant ${ }^{V 600 E} B-R a f$, Mac-1 expression on PMNs was unchanged and significantly less than controls (Fig. 5C).

Collectively, these data indicated that knockdown of mutant ${ }^{V 600 E} B$-Raf inhibited ICAM-1 expression on the surface of melanoma cells as well as Mac-1 up-regulation on PMNs after PMN-melanoma coculture, which in turn retarded PMN-facilitated melanoma extravasation through disruption of ICAM-1- $\beta_{2}$ integrin binding.

\section{Disruption of NF-KB activity following inhibition of mutant ${ }^{V 600 E} B$-Raf reduced IL-8 levels and ICAM-1 expression}

IL-8 protein production requires the activation of nuclear NF- $\mathrm{KB}(13,14)$. NF- $\mathrm{\kappa B}$ has also been reported to induce ICAM-1 expression on melanoma cells (27). Previous studies have implicated Raf kinases in NF-kB signaling $(14,28)$. To investigate whether inhibition of mutant ${ }^{V 600 E} B$-Raf disrupts NF- $\mathrm{\kappa B}$ signaling, thereby reducing IL-8 production from melanoma cells and ICAM-1 expression on melanoma cells, electromobility shift assay was used to measure NF- $\mathrm{KB}$ expression in $1205 \mathrm{Lu}$. Results indicate that NF- $\kappa \mathrm{B}$ expression was reduced by $\sim 50 \%$ following inhibition of mutant ${ }^{V 600 E} B$-Raf (Fig. $6 A$ ). To further evaluate the

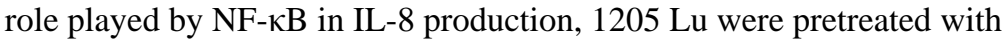
pyrrolidinedithiocarbamate ( $100 \mu \mathrm{mol} / \mathrm{L}$; Calbiochem), a NF- $\kappa \mathrm{B}$ inhibitor, for $1 \mathrm{~h}$ and IL-8 production was measured by ELISA. Results showed that IL-8 levels decreased significantly compared with untreated control (Fig. 6B). In addition, ICAM-1 expression on melanoma cells after pyrrolidinedithiocarbamate treatment was also reduced compared with untreated control (Fig. 6B). Together, these results suggest that interruption of downstream NF- $\mathrm{kB}$ signaling 
following inhibition of mutant ${ }^{V 600 E}$ B-Raf reduced IL-8 production and ICAM-1 expression from melanoma cells to decrease melanoma extravasation.

\section{Discussion}

In this study, a unique strategy using transient siRNA-mediated knockdown of mutant ${ }^{V 600 E} B$-Raf has been used to show that development of metastatic lung tumors was significantly reduced due to the disruption of extravasation through endothelium by targeted inhibition of mutant ${ }^{V 600 E}$ B-Raf. As a result of the inhibition of mutant ${ }^{V 600 E} B$-Raf, the interactions between ICAM- 1 on melanoma and $\beta_{2}$ integrins on PMN as well as the endogenously IL-8 production were disrupted, which in turn reduced melanoma extravasation. This is the first study to identify the role of mutant ${ }^{V 600 E} B$-Raf in PMN-facilitated melanoma extravasation, suggesting a potential mechanism for preventing the metastatic process.

The extravasation of melanoma cells was significantly reduced under static conditions following inhibition of mutant ${ }^{V 600 E} B$-Raf. A previous study has shown that melanoma cell migration to type IV collagen under static condition requires activation of NF- $\mathrm{KB}$ (29). We now show that NF- $\mathrm{KB}$ activation in melanoma cells is reduced after inhibition of mutant ${ }^{V 600 E} B$-Raf, indicating a potential mechanism for reduced extravasation under static conditions. This observation is supported by previous studies showing that mutant ${ }^{V 600 E} B$ $R a f$ renders the mitogen-activated protein pathway constitutively active, thereby eliciting constant activation of downstream signaling components and the corresponding transcription factor substrates including NF- $\mathrm{kB}(30,31)$. Cancer metastasis happens when extravasated cells proliferate and form secondary tumors (32). Our in vitro proliferation experiments showed reduced growth of melanoma cells after knockdown of mutant ${ }^{V 600 E} B$-Raf (data not shown). Therefore, besides the interruption of extravasation after inhibition of the mutant ${ }^{V 600 E} B$-Raf, the decreased proliferation of extravasated cells may also contribute to inhibition of metastasis.

Chemokines or cytokines secreted by tumor cells and/or PMN play important roles in communication between melanomas and PMN. Therefore, disruption of the signals between these cell types could be used for therapeutic advantages (33). IL-8 expression in human melanoma cells correlates with the level of anoxia and the aggressiveness of the melanoma (34). Induction of transcription of IL-8 in these anoxic tumor areas is dependent on NF- $\mathrm{kB}$ activity (35). Consistent with these reports, our results showed that IL-8 secretion from melanoma cells decreased after inhibition of NF- $\mathrm{kB}$. Our results also indicate that knockdown of mutant ${ }^{V 600 E} B-R a f$ significantly decreased the production of IL-8 from melanoma cells alone. It is possible to attribute this observation to the disruption of NF- $\kappa \mathrm{B}$ signaling after inhibition of mutant ${ }^{V 600 E} B$-Raf. In support of this possibility, mutant forms of $B$-Raf have been reported to be able to activate NF- $\kappa \mathrm{B}$ signaling (30). Furthermore, our results indicate that coculture of PMNs and control melanoma cells increased IL-8 production; however, IL-8 production after coculture of PMNs and melanoma following inhibition of mutant ${ }^{V 600 E} B$ Raf was unchanged or even reduced. Previous studies have found that endogenously secreted IL-8 from melanoma cells induced an increase in IL-8 production from PMN after coculture of PMNs and melanoma cells (36). Therefore, reduced IL-8 secretion from melanoma-PMN microenvironment after inhibition of mutant ${ }^{V 600 E} B-R a f$ could be attributed to both reduced IL-8 production from melanoma cells and reduced IL-8 from PMN due to the reduced amount of IL-8 from melanoma cells.

Previous studies have investigated the role of PMNs in mediating melanoma cell adhesion to and extravasation through an endothelial cell layer under flow conditions $(8,11)$. Studies also showed that binding between ICAM- 1 on melanoma and $\beta_{2}$ integrin on PMN is essential for this process. In this study, results showed that inhibition of mutant ${ }^{V 600 E} B$-Raf in melanoma reduced PMN-facilitated melanoma migration significantly. Potential mechanisms were 
investigated and we found that ICAM-1 expression on melanoma cells was reduced after treatment with siRNA against mutant ${ }^{V 600 E} B$-Raf. A possible mechanism causing reduced ICAM-1 expression could be disruption of NF- $\mathrm{KB}$ signaling after inhibition of mutant ${ }^{V 600 E} B-R a f$. In support of this possibility, NF- $\mathrm{KB}$ has been reported to facilitate tumor invasion and metastasis by inducing expression of ICAM-1 on melanoma cells (27). Our results also confirmed that inhibition of NF- $\mathrm{kB}$ reduced ICAM-1 expression on melanoma cells. In addition, we have shown that PMN-melanoma coculture can increase Mac-1 expression on PMNs, which was inhibited after knockdown of mutant ${ }^{V 600 E} B$-Raf. It has also been reported that IL-8 could trigger the functional up-regulation of the ligand binding activity of the $\beta_{2}$ integrins, Mac-1 in particular, on PMNs $(25,37)$. Our results show reduced IL-8 from PMNmelanoma cocultures following inhibition of mutant ${ }^{V 600 E} B-R a f$, possibly explaining the mechanism for reduced Mac-1 expression on PMN after coculture with melanoma following inhibition of mutant ${ }^{V 600 E} B$-Raf. Collectively, those data indicate one mechanism of reduced melanoma extravasation under flow conditions after mutant ${ }^{V 600} B$-Raf inhibition, which is through interruption of the ICAM- $1 / \beta_{2}$ integrin binding mechanism necessary for PMNfacilitated melanoma extravasation.

In conclusion, we showed that targeting mutant ${ }^{V 600 E} B$-Raf reduces melanoma metastasis, which is mediated through reduction of melanoma cell extravasation through the endothelium. Mechanistically, reduced melanoma extravasation following inhibition of mutant ${ }^{V 600 E} B-R a f$ is due to the disruption of downstream NF- $\mathrm{\kappa B}$ signaling that causes reduced endogenous IL-8 production and the disruption of ICAM- $1 / \beta_{2}$ integrin binding that is essential for PMNfacilitated melanoma extravasation. Therefore, this study suggests that targeting mutant ${ }^{V 600 E} B$-Raf signaling may offer a potential mechanism for therapeutic inhibition of NF$\kappa \mathrm{B}$ functioning in melanoma.

\section{Acknowledgments}

Grant support: NIH grants CA-97306 (C. Dong) and M01-RR-10732 (GCRC), National Science Foundation grant BES-0138474 (C. Dong), Johnson \& Johnson Innovative Technology Research Seed Grant Program (C. Dong and G.P. Robertson), Grace Woodward Grants in Engineering and Medicine (C. Dong and G.P. Robertson), The American Cancer Society grant RSG-04-053-01-GMC (G.P. Robertson), and The Melanoma Research Foundation and The Foreman Foundation for Melanoma Research (G.P. Robertson).

\section{References}

1. Brose M, Volpe P, Feldman M. BRAF and RAS mutations in human lung cancer and melanoma. Cancer Res 2002;62:6997-7000. [PubMed: 12460918]

2. Davies H, Bignell G, Cox C, et al. Mutations of the BRAF gene in human cancer. Nature 2003;417:949954. [PubMed: 12068308]

3. Gorden A, Osman I, Gai W, et al. Analysis of B-Raf and N-Ras mutations in metastatic melanoma tissues. Cancer Res 2003;63:3955-3957. [PubMed: 12873990]

4. Pollock P, Harper U, Hansen K. High frequency of BRAF mutations in nevi. Nat Genet 2003;33:1920. [PubMed: 12447372]

5. Mercer K, Pritchard C. Raf proteins and cancer: $B$-Raf is identified as a mutational target. Biochim Biophys Acta 2003;1653:25-40. [PubMed: 12781369]

6. Chang F, Steelman L, Shelton J. Regulation of cell cycle progression and apoptosis by the Ras/Raf/ MEK/ERK pathway. Int J Oncol 2003;22:469-480. [PubMed: 12579299]

7. Yazdi A, Palmedo G, Flaig M, et al. Mutations of the BRAF gene in benign and malignant melanocytic lesions. J Invest Dermatol 2003;121:1160-1162. [PubMed: 14708620]

8. Slattery M, Dong C. Neutrophils influence melanoma adhesion and migration under flow conditions. Int J Cancer 2003;106:713-722. [PubMed: 12866031]

9. Wu Q, Wang J, Condron C, Bouchier-Hayer D, Redmond H. Human neutrophils facilitate tumor cell transendothelial migration. Am J Physiol Cell Physiol 2001;280:C814-C822. [PubMed: 11245598] 
10. Miele M, Bennett C, Miller B, Welch D. Enhanced metastatic ability of TNF-treated maliganant melanoma cells is reduced by intercellular adhesion molecule-1 (ICAM-1) antisense oligonucleotides. Exp Cell Res 1994;214:231-241. [PubMed: 7915992]

11. Liang S, Slattery M, Dong C. Shear stress and shear rate differentially affect the multi-step process of leukocyte-facilitated melanoma adhesion. Exp Cell Res 2005;310:282-292. [PubMed: 16154563]

12. Slattery M, Liang S, Dong C. Distinct role of hydrodynamic shear in leukocyte-facilitated tumor cell extravasation. Am J Physiol Cell Physiol 2005;288:C831-C839. [PubMed: 15601752]

13. Dhawan P, Richmond A. A novel NF- $\mathrm{BB}$-inducing kinase-MAPK signaling pathway up-regulates NF- $\kappa$ B activity in melanoma cells. J Biol Chem 2002;277:7920-7928. [PubMed: 11773061]

14. Richmond A. NFkB, chemokine gene transcription and tumor growth. Nat Rev Immunol 2002;2:664674. [PubMed: 12209135]

15. Stahl JM, Sharma A, Cheung M, et al. Deregulated Akt3 activity promotes development of malignant melanoma. Cancer Res 2004;64:7002-7010. [PubMed: 15466193]

16. Miller C, Cheung M, Sharma A. Method of mutation analysis may contribute to discrepancies in reports of BRAF mutation frequencies in melanocytic neoplasms. J Invest Dermatol 2004;123:990992. [PubMed: 15482489]

17. Gopalan P, Smith C, Lu H, Berg E, Mcintire L, Simon S. PMN CD18-dependent arrest on ECAM-1 in shear flow can be activated through L-selectin. J Immunol 1997;158:367-375. [PubMed: 8977212]

18. Sharma A, Tran AM, Liang S, et al. Targeting mitogen-activated protein kinase/extracellular signalregulated kinase kinase in the mutant (V600E) $B$-Raf signaling cascade effectively inhibits melanoma lung metastases. Cancer Res 2006;66:8200-8209. [PubMed: 16912199]

19. Sharma A, Trivedi N, Tuveson D, Smith C, Robertson G. Mutant V599E B-Raf regulates growth and vascular development of malignant melanoma tumors. Cancer Res 2005;65:2412-2421. [PubMed: 15781657]

20. Dong C, Slattery M, Rank B, You J. In vitro characterization and micromechanics of tumor cell chemotactic protrusion, locomotion, and extravasation. Ann Biomed Eng 2002;30:344-355. [PubMed: 12051619]

21. Dong C, Aznavoorian S, Liotta L. Two phases of pseudopod protrusion in tumor cells revealed by a micropipette. Microvasc Res 1994;47:55-67. [PubMed: 8022314]

22. You J, Aznavoorian S, Liotta AL, Dong C. Responses of tumor cell pseudopod protrusion to changes in medium osmolality. J Cell Physiol 1995;167:156-163. [PubMed: 8698833]

23. Shevde L, Welch D. Metastasis suppressor pathways - an evolving paradigm. Cancer Lett 2003;198:1-20. [PubMed: 12893425]

24. Dong C, Slattery M, Liang S, Peng H. Melanoma cell extravasation under flow conditions is modulated by leukocytes and endogenously produced interleukin 8. Mol Cell Biomech 2006;2:145159. [PubMed: 16708476]

25. Detmers P, Lo S, Olsen-Egbert E, Walz A, Baggiolini M, Cohn Z. Neutrophil-activating protein 1/ interleukin 8 stimulates the binding activity of the leukocyte adhesion receptors CD11b/CD18 on human neutrophils. J Exp Med 1990;171:1155-1162. [PubMed: 1969919]

26. Detmers P, Powell D, Walz A, Clark-Lewis I, Baggiolini M, Cohn Z. Differential effects of neutrophilactivating peptide $1 /$ IL- 8 and its homologous on leukocyte adhesion and phagocytosis. J Immunol 1991;147:4211-4217. [PubMed: 1721641]

27. Stople A, van de, Caldenhoven E, Stade BG, et al. 12-O-Tetradecanoylphorbol-13-acetate- and tumor necrosis factor $\alpha$-mediated induction of intercellular adhesion molecule- 1 is inhibited by dexamethasone. J Biol Chem 1994;269:6185-6192. [PubMed: 7907090]

28. Baumann B, Weber C, Troppmair J, et al. Raf induces NF- $\kappa$ B by memebrane shuttle kinase MEKK1, a signaling pathway critical for transformation. Proc Natl Acad Sci US A 2000;97:4615-4620.

29. Hodgson L, Henderson A, Dong C. Melanoma cell migration to type IV collagen requires activation of NF-kB. Oncogene 2003;22:98-108. [PubMed: 12527912]

30. Ikenoue T, Hikiba Y, Kanai F, et al. Different effects of point mutations within the B-Raf glycinerich loop in colorectal tumors on mitogen-activated proetin/extracellular signal-regulated kinase kinase/extracellular signal-regulated kinase and nuclear factor nB pathway and cellular transformation. Cancer Res 2004;64:3428-3435. [PubMed: 15150094] 
31. Ikenoue T, Hikiba Y, Kanai F, et al. Analysis of mutations within the kinase activation segment of B-Raf in human colorectal tumors. Cancer Res 2003;63:8132-8137. [PubMed: 14678966]

32. Laud K, Kannengiesser C, Avril M, et al. BRAF as a melanoma susceptibility candidate gene? Cancer Res 2003;63:3061-3065. [PubMed: 12810628]

33. Frederick M, Clayman G. Chemokines in cancer. Expert Rev Mol Med 2001;2001:1-81. [PubMed: 14585143]

34. Kunz M, Hartmann A, Flory E, et al. Anoxia-induced up-regulation of interleukin-8 in human malignant melanoma. Am J Pathol 1999;155:753-763. [PubMed: 10487833]

35. Wang W, Abbruzzese J, Evans D, Larry L, Cleary K, Chiao P. The nuclear factor- $\kappa \mathrm{B}$ RelA transcription factor is consititutively activated in human pancreatic adeno-carcinoma cells. Clin Cancer Res 1999;5:119-127. [PubMed: 9918209]

36. Peng HH, Liang S, Henderson AJ, Dong C. Regulation of interleukin-8 expression in melanomastimulated neutrophil inflammatory response. Exp Cell Res 2007;313:551-559. [PubMed: 17141217]

37. Diamond M, Springer T. The dynamic regulation of integrin adhesiveness. Curr Biol 1994;4:506517. [PubMed: 7922371] 


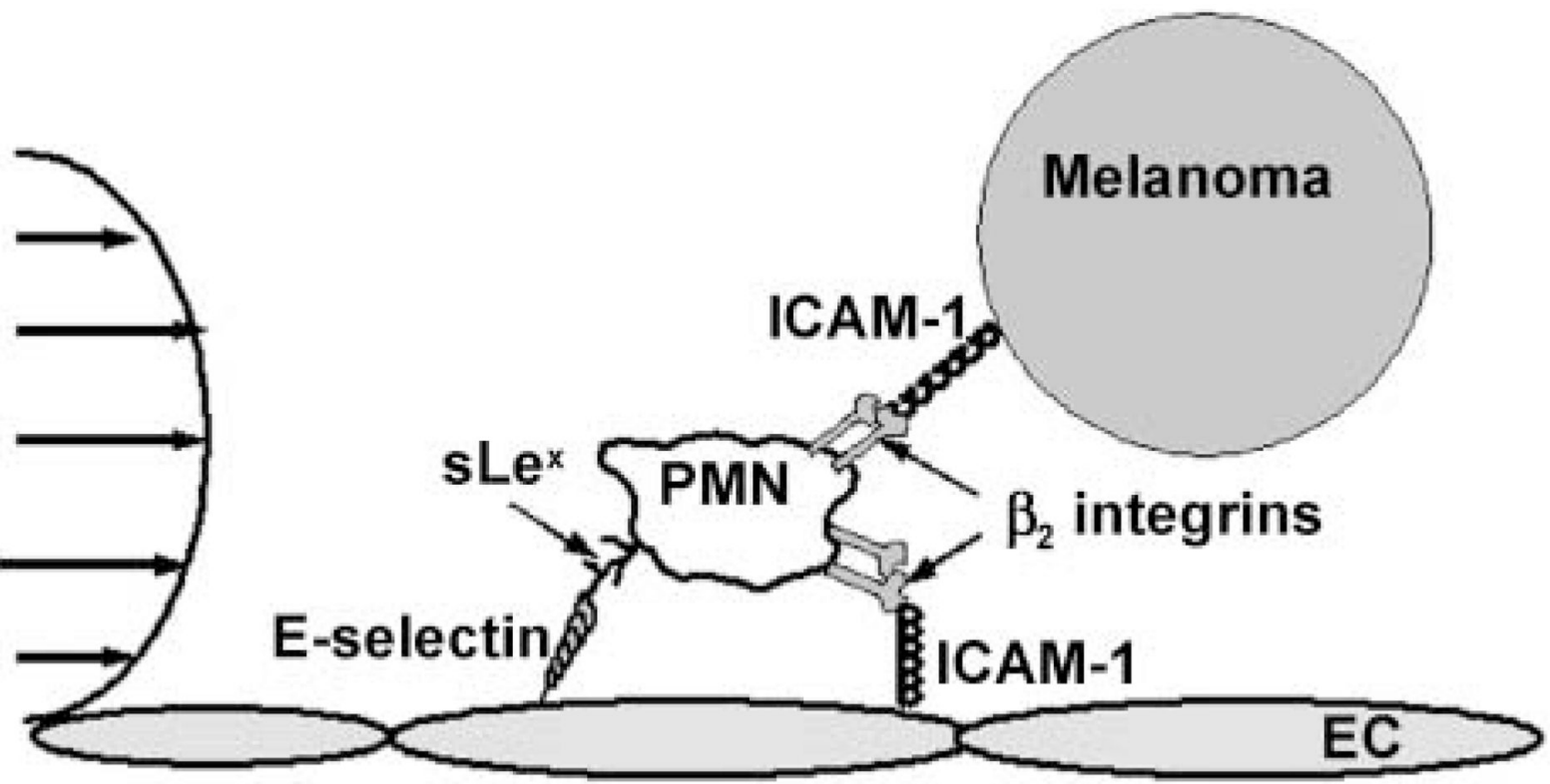

Figure 1. Schematic of endothelium-PMN-melanoma interactions under flow conditions PMNs use selectin receptors to form rolling attachments along the vessel wall and $\beta_{2}$ integrins to anchor firmly through $\beta_{2}$ integrin-ICAM-1 binding. In contrast, melanoma cells do not express $\beta_{2}$ integrins or sialylated molecules at levels needed to effectively adhere to the endothelium $(E C)$ under flow conditions. However, melanoma cells also express ICAM-1; a collision with PMNs in a shear flow could result in the formation of aggregates and then bring melanoma cells close to endothelium. 


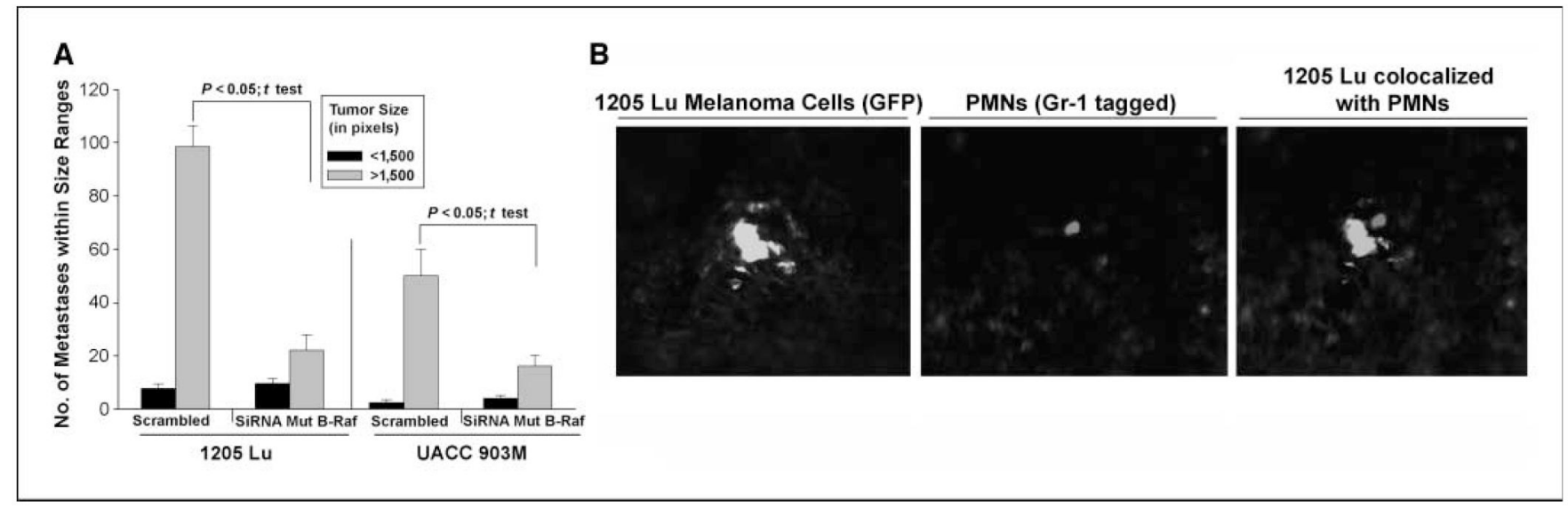

Figure 2. In vivo lung metastasis study

$A$, siRNA-mediated inhibition of ${ }_{V 600 E} B$-Raf inhibits melanoma lung metastasis. Left, siRNAmediated inhibition of mutant ${ }_{V 600 E} B-R a f$ in $1205 \mathrm{Lu}$ cells reduced formation of melanoma lung metastases. Right, siRNA-mediated inhibition of mutant ${ }^{V 60 E_{B}}$-Raf in UACC 903M cells reduced formation of melanoma lung metastases. Number of tumors within particular size ranges $(<1,500$ or $>1,500$ pixels $)$ were quantified in a minimum of six fields per lung from 5 to 10 animals. Columns, mean; bars, SE. B, colocalization of melanoma cells and mouse PMNs in vivo. Left, green GFP-tagged melanoma cells. Middle, red phycoerythrin-labeled PMNs. Right, colocalization of green GFP-tagged melanoma cells and red phycoerythrin-labeled PMNs. 

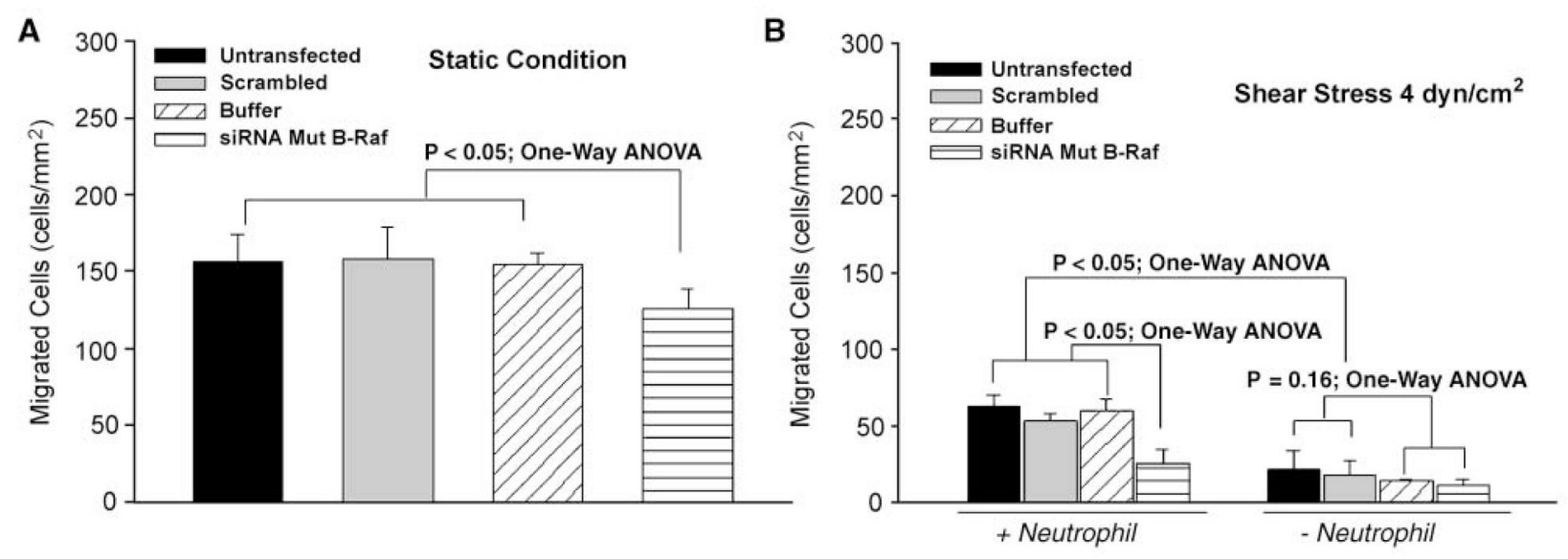

Figure 3. Inhibiting ${ }^{V 600 E} B$-Raf reduced melanoma cell extravasation in vitro.

$A$, under static condition, knockdown of mutant ${ }^{V 600 E} B$-Raf significantly reduced melanoma migration toward chemoattractant compared with control cases (untransfected melanoma and melanoma nucleofected with buffer only or scrambled siRNA). $B$, PMN enhanced melanoma extravasation under flow condition $\left(4 \mathrm{dyn} / \mathrm{cm}^{2}\right)$. Inhibition of mutant ${ }^{V 600 E} B$-Raf reduced PMN-facilitated melanoma extravasation significantly compared with control cases.

Columns, mean; bars, SE. 

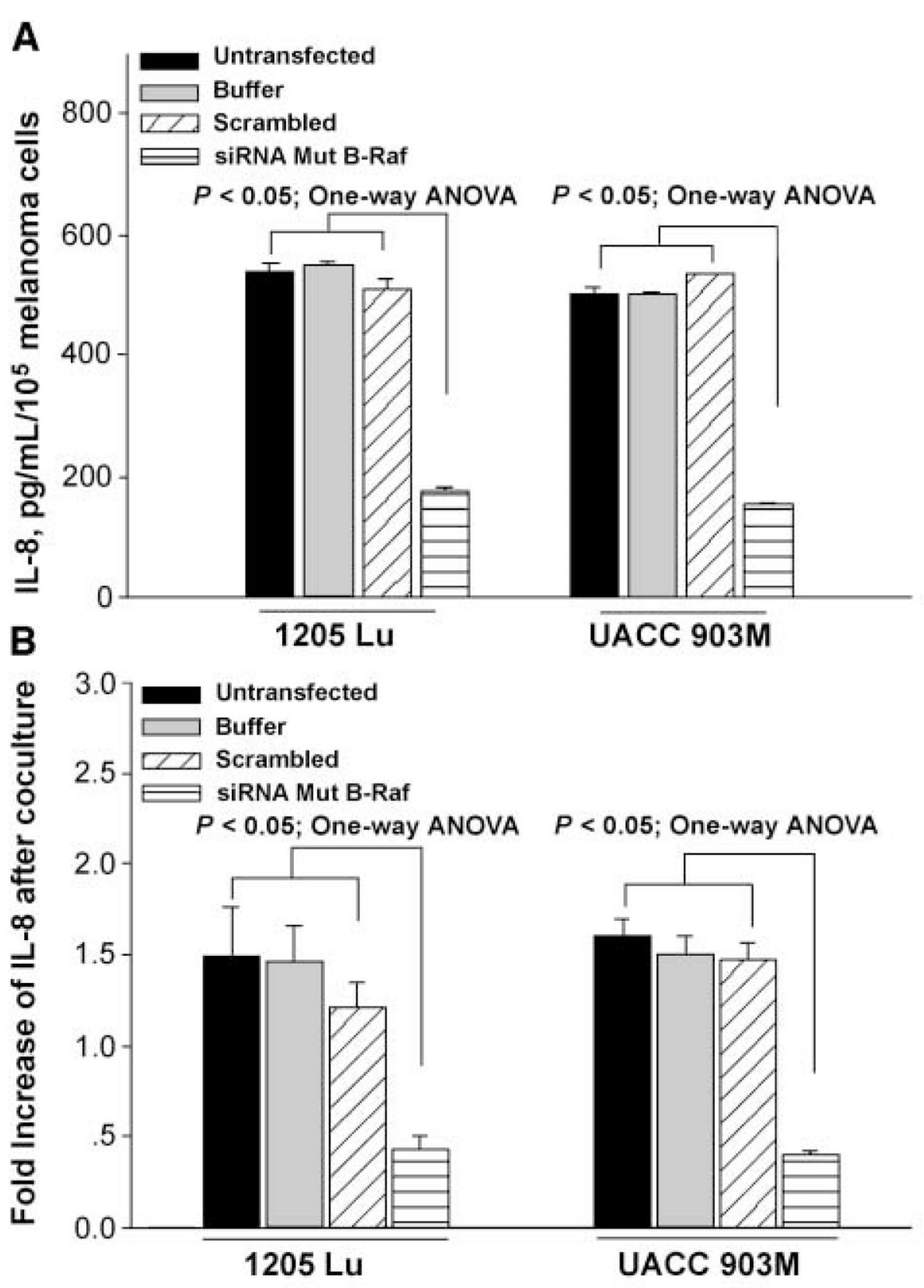

Figure 4. Inhibiting ${ }^{V 600} E_{B-R a f}$ reduced IL-8 production

$A$, inhibition of mutant $V 600 E B$-Raf significantly reduced IL-8 production from melanoma cells (1205 Lu and UACC 903M) cultured alone compared with the control melanoma cells (untransfected melanoma and melanoma nucleofected with buffer only or scrambled siRNA). Columns, mean; bars, SE. B, IL-8 production from tumor microenvironment (including both PMN and melanoma cells) increased after PMN coculture with control melanoma cells ( 1.5fold), whereas IL-8 production either remained the same or was even reduced after PMN coculture with melanoma cells treated with siRNA against mutant ${ }^{V 600 E} B$-Raf. Values were normalized to the summed background level of PMN and melanoma cells cultured alone. Columns, mean; bars, SE. 


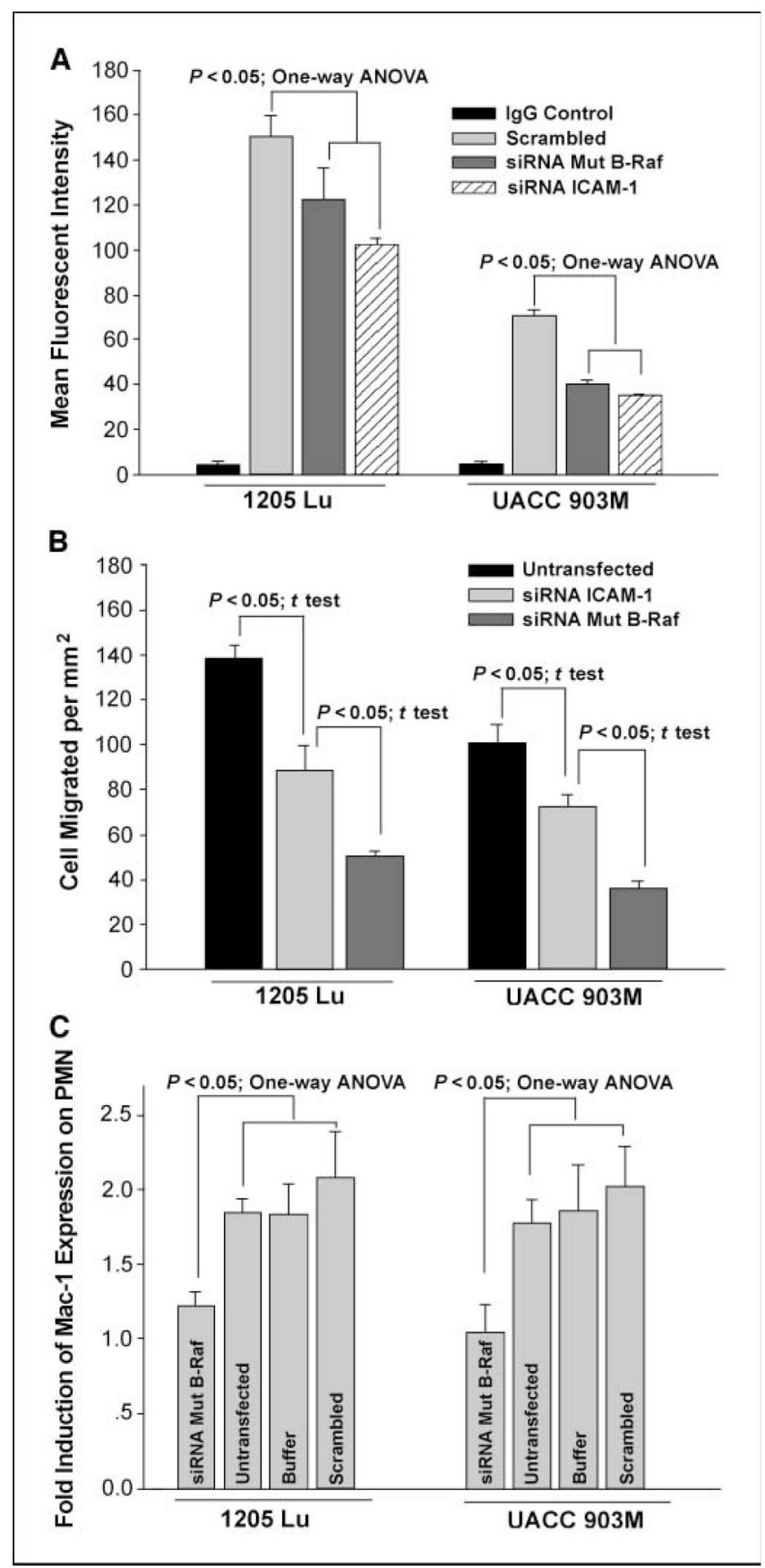

Figure 5. Disruption of ICAM-1/ $\beta_{2}$ integrin binding mechanism in PMN-mediated melanoma extravasation in vitro after knockdown of mutant ${ }^{V 600 E} B$-Raf

$A$, ICAM-1 expression on melanoma cells (1205 Lu and UACC 903M) was reduced after knockdown of mutant ${ }^{V 600 E} B-R a f$ and ICAM-1 using siRNA. $B$, knockdown of mutant ${ }^{V 600}$ B-Raf and ICAM-1 inhibited PMN-mediated melanoma extravasation.

Columns, mean; bars, SE. C, Mac-1 expression on PMN after coculture with melanoma cells (1205 Lu and UACC 903M). Mac-1 expression on PMN increased significantly after PMN coculture with control melanoma cells (untransfected melanoma and melanoma nucleofected with buffer only or scrambled siRNA). However, the coculture between PMN and melanoma cells treated with siRNA against ${ }^{V 600 E} B$-Raf did not significantly increase Mac-1 expression 
on PMN. Values were normalized to background Mac-1 expression on PMN alone. Columns, mean; bars, SE. 


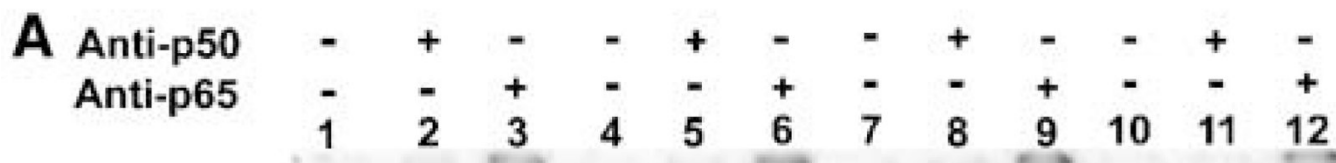

\section{$\mathrm{NF}-\mathrm{kB}$ super shift}

B

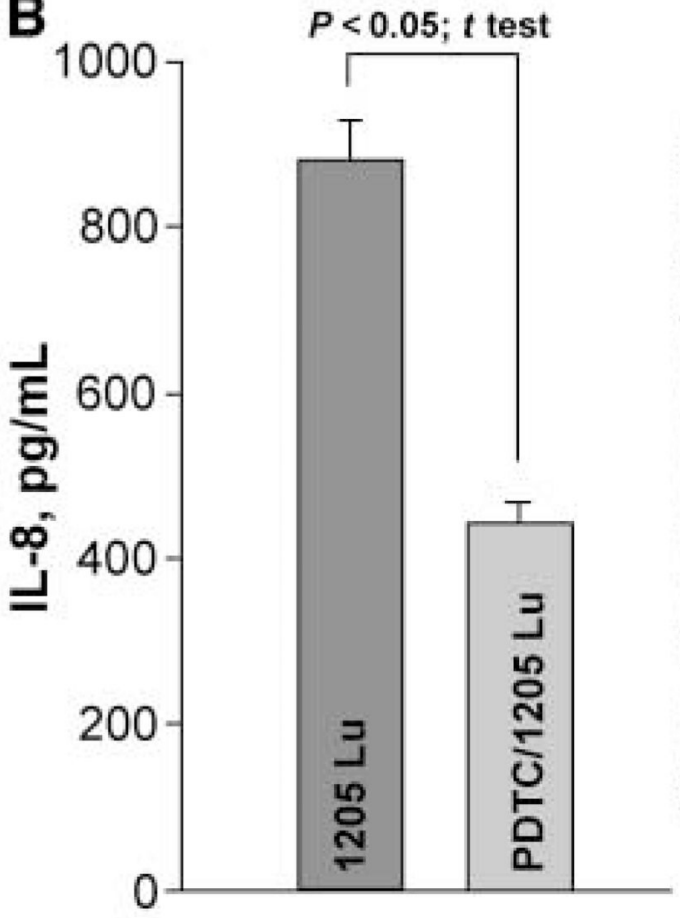

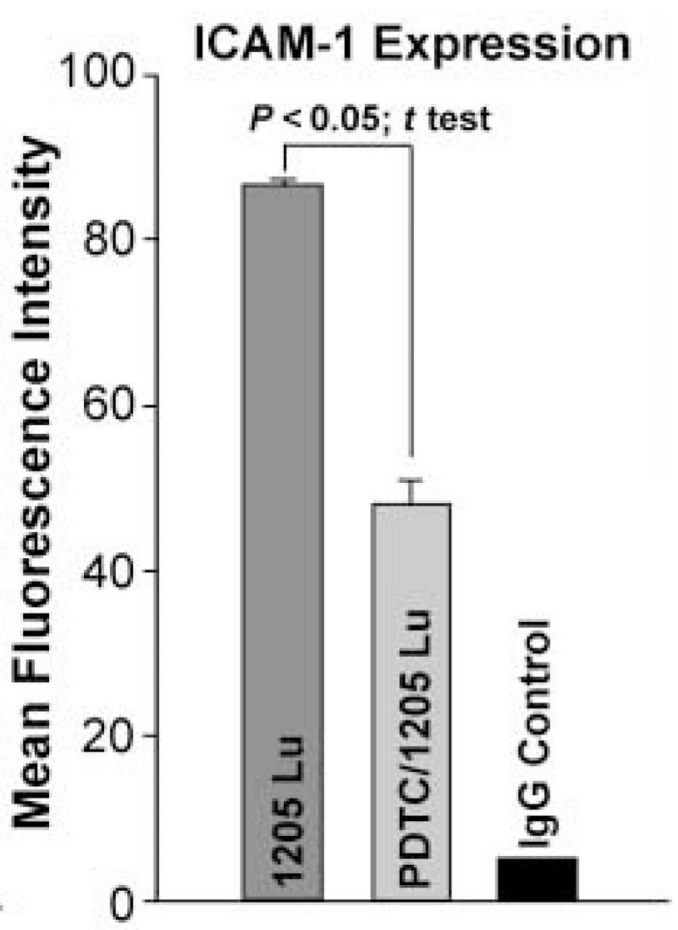

Figure 6. Disruption of NF-кB activity after inhibition of mutant ${ }^{V 600 E} B-R a f$ $A, \mathrm{NF}-\kappa \mathrm{B}$ activity is reduced after inhibition of mutant ${ }^{V 600 E} \mathrm{~B}-$ Raf in $1205 \mathrm{Lu}$ (lane 10) compared with control cases (lane 1, untransfected $1205 \mathrm{Lu}$; lane 4, 1205 Lu nucleofected with scrambled siRNA; or lane 7, buffer only). The complexes were supershifted by polyclonal antibodies against p50 (lanes 2, 5, 8, and 11) and p65 (lanes 3, 6, 9, and 12). B, pyrrolidinedithiocarbamate (PDTC) treatment reduced IL-8 secretion and ICAM-1 expression on melanoma cells. $1205 \mathrm{Lu}$ cells were treated with pyrrolidinedithiocarbamate $(100 \mu \mathrm{mol} / \mathrm{L})$ for $1 \mathrm{~h}$. After washing twice, $1205 \mathrm{Lu}$ cells were cultured using fresh culture media. Left, supernatant after $4 \mathrm{~h}$ was collected and IL-8 secretion was detected by ELISA. Right, ICAM-1 expression on $1205 \mathrm{Lu}$ was examined by flow cytometry. 